\title{
Some Construction Methods of Optimum Chemical Balance Weighing Designs III
}

\author{
Rashmi Awad*, Shakti Banerjee \\ School of Statistics, Devi Ahilya University, Indore, India \\ Email: *awad.rashmi@gmail.com, shaktibn@yahoo.com
}

Received 24 November 2015; accepted 15 February 2016; published 18 February 2016

Copyright (C) 2016 by authors and Scientific Research Publishing Inc.

This work is licensed under the Creative Commons Attribution International License (CC BY). http://creativecommons.org/licenses/by/4.0/

c) (i) Open Access

\begin{abstract}
Methods of constructing the optimum chemical balance weighing designs from symmetric balanced incomplete block designs are proposed with illustration. As a by-product pairwise efficiency and variance balanced designs are also obtained.
\end{abstract}

\section{Keywords}

Balanced Incomplete Block Design, Symmetric Balanced Incomplete Block Design, Ternary Balanced Block Design, Variance Balanced Design, Efficiency Balanced Design, Nested Balanced Incomplete Block Design, Weighing Design, Chemical Balance Weighing Design, Optimum Chemical Balance Weighing Design

\section{Introduction}

Originally Yates (see [1]) gave the concept of weighing design. Afterward his work was formulated by Hotelling (see [2]) and he gave the condition of attaining the lower bound by each of the variance of the estimated weights. Many statisticians did prominent work in obtaining optimum weighing designs (see [3]-[7]). In recent years, different methods of constructing the optimum chemical balance weighing designs; using the incidence matrices of known balanced incomplete block designs, balanced bipartite block designs, ternary balanced block designs and group divisible designs have been given in the literature (see [8]-[11]).

Construction methods of obtaining optimum chemical balance weighing designs using the incidence matrices of symmetric balanced incomplete block designs have been given by Awad et al. [12]-[14]; some pairwise balanced designs are also been obtained which are efficiency as well as variance balanced. In this paper; some other new construction methods of obtaining optimum chemical balance weighing designs using the incidence matrices of known symmetric balanced incomplete block designs are propose. Some more pairwise efficiency as

${ }^{*}$ Corresponding author. 
well as variance balanced designs are also been proposed.

Let us consider a block design in which $v$ treatments arranged in $b$ blocks and elements of the incidence matrix $N$ are denoted by $\left[n_{i j}\right]$, for all $i=1,2, \cdots, v ; j=1,2, \cdots, b$, such that the $j^{\text {th }}$ block contains $k_{j}$ experimental units and the $i^{\text {th }}$ treatment appears $r_{i}$ times in the entire design. A balanced block design is said to be (a) binary when $n_{i j}=0$ or $1, \forall i=1,2, \cdots, v ; j=1,2, \cdots, b$. Otherwise, it is said to be nonbinary (see [7]); (b) ternary if $n_{i j}=0,1$ or $2, \forall i=1,2, \cdots, v ; j=1,2, \cdots, b$, and it has parameters $v, b, \rho_{1}, \rho_{2}, r, k, \Lambda$; where $\rho_{1}, \rho_{2}$ are the number of times 1,2 occurs in the incidence matrix, respectively (see [15]); (c) generalized binary if $n_{i j}=0$ or $x, \forall i=1,2, \cdots, v ; j=1,2, \cdots, b$ and some positive integer $x(>1)$, and it has parameters $v, b, r, k, \Lambda$ (see [16]).

A balanced incomplete block design is an arrangement of $v$ symbols (treatments) into $b$ sets (blocks) such that (1) each block contains $k(<v)$ distinct treatments; (2) each treatment appears in $r(>\lambda)$ different blocks and; (3) every pair of distinct treatments appears together in exactly $\lambda$ blocks. Here, the parameters of balanced incomplete block design $(v, b, r, k, \lambda)$ are related by the following relations

$$
v r=b k, r(k-1)=\lambda(v-1) \text { and } b \geq v \text { (Fisher's Inequality) }
$$

A balanced incomplete block design is said to be symmetric if $b=v$ ( consequently, $r=k$ ). In this case, incidence matrix $N$ is a square matrix i.e. $N^{\prime}=N$. In case of symmetric balanced incomplete block design any two sets have $\lambda$ symbols in common.

Balancing of design in various senses has been given in the literature (see [17] [18]). In this paper, we consider the balanced design of the following types: 1) variance balanced block designs; 2) efficiency balanced block designs and; 3) pairwise balanced block designs.

1) A block design is said to be variance balanced if and only if its C-matrix, $R-N K^{-1} N$, satisfies $R-N K^{-1} N=\psi\left[I_{v}-(1 / v) 1_{v} 1_{v}^{\prime}\right]$, for some constant $\psi$ (see [19]-[22]); where $\psi$ is the unique nonzero eigen value of the matrix $C$ with the multiplicity $(v-1), I_{v}$ is the $v \times v$ identity matrix.

2) A block design is said to be efficiency balanced if $R^{-1} N K^{-1} N^{\prime}=\mu I_{v}+[(1-\mu) / n] 1_{v} r^{\prime}$, for some constant $\mu$ (see [20]-[22]); where $\mu$ is the unique non zero eigen value with multiplicity $(v-1)$. For the EB block design $N$, the information matrix is given as $C=(1-\mu)\left(R-(1 / n) \underline{r} \underline{r}^{\prime}\right)$; see [23]

3) A block design is said to be pairwise balanced if $\sum_{j=1}^{b} n_{i j} n_{i^{\prime} j}=\Lambda$ (a constant) for all $i$, $i^{\prime}$; where $i \neq i^{\prime}$ and $i, i^{\prime} \in 1,2, \cdots, v$. A pairwise balanced block design is said to be binary if $n_{i j}=0$ or 1 only, for all $i, j$ and it has parameters $v, b, \underline{r}, \underline{k}, \Lambda$ (= $\lambda$, say) (in this case, when $\underline{r}=r 1_{v}$ and $\underline{k}=k 1_{b}$, it is a BIB design with parameters $v, b, \underline{r}, \underline{k}, \bar{\lambda})$.

A design is said to form a nested structure, when there are two sources of variability and one source is nested within other. Preece (see [24]) introduced a class of nested BIB designs with $v$ treatments, each replicated $r$ times, with two systems of blocks, such that (a) the second system nested within the first, with each block from the first system (called super blocks) containing exactly " $m$ " blocks from the second system (called sub-blocks). (b) Ignoring the sub-blocks, leaves a BIB design with parameters $v, b^{*}, r, k^{*}, \lambda^{*}$. (c) Ignoring the superblocks, leaves a BIB design with parameters $v, b_{*}, r, k_{*}, \lambda_{*}$. The $v, b^{*}, b_{*}, r, k^{*}, k_{*}, \lambda^{*}, \lambda_{*}$ and $m$ are called the parameters of a nested BIB design. The parameters satisfy the following conditions:

$$
\begin{aligned}
& v r=b^{*} k^{*}=b^{*} m k_{*}=b_{*} k_{*} \\
& r\left(k^{*}-1\right)=\lambda^{*}(v-1) \\
& r\left(k_{*}-1\right)=\lambda_{*}(v-1)
\end{aligned}
$$

so that

$$
(v-1)\left(\lambda^{*}-m \lambda_{*}\right)=(m-1) r
$$

The following additional notations are used $\underline{k}=\left[k_{1} k_{2} \cdots k_{b}\right]^{\prime}$ is the column vector of block sizes, $\underline{r}=\left[r_{1} r_{2} \cdots r_{v}\right]^{\prime}$ is the column vector of treatment replication, $K_{b \times b}=\operatorname{diag}\left[k_{1} k_{2} \cdots k_{b}\right], R_{v \times v}=\operatorname{diag}\left[r_{1} r_{2} \cdots r_{v}\right]$, $\sum_{i=1}^{v} r_{i}=\sum_{j=1}^{b} k_{j}=n$ is the total number of experimental units, with this $N 1_{b}=\underline{r}$ and $N^{\prime} 1_{v}=\underline{k}$, Where $1_{a}$ is the $a \times 1$ vector of ones. Furthermore $\mu$ represents the loss of information, i.e., $1-\mu$ represents an effi- 
ciency of the design, $(1 / \psi)$ represents the variance of any normalized contrast in the intra block analysis (see [20] [23] [25] [26]).

For given $p$ objects to be weighted in groups in $\mathrm{n}$ weightings, a weighing designs consists of $n$ groupings of the $\mathrm{p}$ objects and the least square estimates of the weight of the objects can be obtained by the usual methods when $n \geq p$. Using matrix notations the general linear model can be written as:

$$
\underline{Y}=X \underline{w}+\underline{e}
$$

where $\underline{Y}$ is $n \times 1$ column vector of the weights of the objects, $\underline{w}$ is the $p \times 1$ column vector of the unknown weights of objects and $\underline{e}$ is $n \times 1$ vector of error components in the different observations such that $E(\underline{e})=0_{n}$ and $E\left(\underline{e e^{\prime}}\right)=\sigma^{2} I_{n}$. Also $X=\left(x_{i j}\right), \quad(i=1,2, \cdots, n ; j=1,2, \cdots, p)$ is the $n \times p$ matrix of known quantities called design matrix whose entries are $+1,-1$ or 0 . Let matrix $X$ takes the values as:

$$
x_{i j}= \begin{cases}+1 & \text { if the } j^{\text {th }} \text { object is in the left pan in the } i^{t^{t h}} \text { weighing } \\ -1 & \text { if the } j^{\text {th }} \text { object is in the right pan in the } i^{\text {th }} \text { weighing } \\ 0 & \text { if the } j^{\text {th }} \text { object is not in the } i^{\text {th }} \text { weighing }\end{cases}
$$

The normal equations for estimating $\underline{w}$ are as follows:

$$
X^{\prime} X \underline{\hat{w}}=X^{\prime} \underline{Y}
$$

where $\underline{\hat{w}}$ is the vector of the weights estimated by the least squares method.

Singularity or non-singularity of a weighing design depends on whether the matrix $X^{\prime} X$ is singular or nonsingular, respectively. When $X$ is of full rank, then it is obvious that the matrix $X^{\prime} X$ is non-singular. Then in this case the least squares estimate of $\underline{w}$ is given by

$$
\underline{\hat{w}}=\left(X^{\prime} X\right)^{-1} X^{\prime} \underline{Y}
$$

and the variance-covariance matrix of $\hat{w}$ is

$$
\operatorname{Var}(\underline{\hat{w}})=\sigma^{2}\left(X^{\prime} X\right)^{-1}
$$

A weighing design is said to be the chemical balance weighing design if the objects are placed on two pans in a chemical balance. In a chemical balance weighing design, the elements of design matrix $X=\left(x_{i j}\right)$ takes the values as +1 if the $j^{\text {th }}$ object is placed in the left pan in the $i^{\text {th }}$ weighing, -1 if the $j^{\text {th }}$ object is placed in the right pan in the $i^{\text {th }}$ weighing and 0 if the $j^{\text {th }}$ object is not weighted in the $i^{\text {th }}$ weighing.

Hotelling (see [2]) has shown that the precision of the estimates of the weight of the object increases further by placing in the other pan of the scale those objects not included in the weighing and thus using two pan chemical balance. He proved that if $\mathrm{n}$ weighing operations have been done to determine the weight of $p=n$ objects, the minimum attainable variance for each of the estimated weights in this case is $\sigma^{2} / n$ and he also shown that each of the variance of the estimated weights attains the minimum if and only if $\left(X^{\prime} X\right)=n I_{p}$. A design satisfying this condition is called an optimum chemical balance weighing design.

\section{Variance Limit of Estimated Weights}

Ceranka et al. (see [8]) studied the problem of estimating individual weights of objects, using a chemical balance weighing design under the restriction on the number of times in which each object is weighed. A lower bound for the variance of each of the estimated weights from this chemical balance weighing design is obtained and a necessary and sufficient condition for this lower bound to be attained was given. Then Ceranka et al. (see [8]) proved the following theorem:

Theorem 2.1. For any $n \times p$ matrix $X$, of a nonsingular chemical balance weighing design, in which maximum number of elements equal to -1 and 1 in columns is equal to $\mathrm{m}$, where $m=\max \left[m_{1}, m_{2}, \cdots, m_{p}\right]$ (where $m_{j}$ be the number of elements equal to -1 and 1 in $j^{\text {th }}$ column of matrix $X$ ). Then each of the variances of the estimated weights attains the minimum if and only if

$$
\left(X^{\prime} X\right)=m I_{p}
$$

Also a nonsingular chemical balance weighing design is said to be optimal for the estimating individual 
weights of objects; if the variances of their estimators attain the lower bound given by,

$$
\operatorname{Var}(\hat{w})=\sigma^{2} / m ; j=1,2, \cdots, p
$$

Preece [24] introduced a class of nested BIB designs. Using this concept Banerjee; (see [27]-[29]) constructed the nested EB as well as VB designs, where the sub-blocks form ternary design $D$ with parameters $\left(v, b, \rho_{1 i}\right.$, $\left.\rho_{2 i}, r, k, \Lambda\right)$ while the super-blocks form generalized binary design $D$ with parameters $(v, b, r, k, \Lambda)$; where $i$ $=1,2$.

Proposition 2.2. Existence of BIB design $D$ with parameters $(v, b, r, k, \lambda)$ implies the existence of a nested $E B$ as well as VB design. The sub-blocks form an EBT as well as VBT design with parameters

$$
\begin{gathered}
v^{* 1}=v, \quad b^{* 1}=2 \lambda\left(\begin{array}{l}
v \\
2
\end{array}\right), \quad \rho_{11}^{\prime}=r(k-1)(k-2), \quad \rho_{21}^{\prime}=r(k-1), \quad r^{* 1}=r k(k-1), \quad k^{* 1}=k, \\
\Lambda^{* 1}=\lambda(k+1)(k-2), \quad \mu^{* 1}=\left[v^{* 1}\left(k^{* 1}+2\right)-\left(k^{* 1}\right)^{2}\right] /\left[\left(k^{* 1}\right)^{2}\left(v^{* 1}-1\right)\right], \quad \psi^{* 1}=\left[v^{* 1} \Lambda^{* 1} / k^{* 1}\right]
\end{gathered}
$$

while the super-blocks form a generalized binary EB as well as VB design with parameters

$$
\begin{gathered}
v_{* 1}=v, \quad b_{* 1}=\lambda\left(\begin{array}{l}
v \\
2
\end{array}\right), \quad r_{* 1}=r k(k-1), k_{* 1}=2 k, \Lambda_{* 1}=2 \lambda k(k-1), \\
\mu_{* 1}=\left[\left(2 v_{* 1}-k_{* 1}\right) / k_{* 1}\left(v_{* 1}-1\right)\right], \quad \psi_{* 1}=\left[v_{* 1} \Lambda_{* 1} / k_{* 1}\right]
\end{gathered}
$$

Proposition 2.3. Existence of BIB design $D$ with parameters $(v, b, r, k, \lambda)$ implies the existence of a nested $E B$ as well as VB design. The sub-blocks form an EBT as well as VBT design with parameters

$$
\begin{gathered}
v^{* 2}=v, \quad b^{* 2}=3 \lambda\left(\begin{array}{l}
v \\
2
\end{array}\right), \quad \rho_{12}^{\prime}=r(k-1)(3 k-4) / 2, \quad \rho_{22}^{\prime}=r(k-1), \quad r^{* 2}=3 r k(k-1) / 2, \quad k^{* 2}=k, \\
\Lambda^{* 2}=\lambda\left(3 k^{2}-3 k-4\right) / 2, \quad \mu^{* 2}=\left[4 v^{* 2}+3 k^{* 2}\left(v^{* 2}-k^{* 2}\right)\right] /\left[3\left(k^{* 2}\right)^{2}\left(v^{* 2}-1\right)\right], \quad \psi^{* 2}=\left[v^{* 2} \Lambda^{* 2} / k^{* 2}\right]
\end{gathered}
$$

while the super-blocks form a generalized binary EB as well as VB design with parameters

$$
\begin{gathered}
v_{* 2}=v, \quad b_{* 2}=\lambda\left(\begin{array}{l}
v \\
2
\end{array}\right), \quad r_{* 2}=[3 r k(k-1)] / 2, \quad k_{* 2}=3 k, \Lambda_{* 2}=[9 \lambda k(k-1)] / 2, \\
\mu_{* 2}=\left[\left(3 v_{* 2}-k_{* 2}\right) / k_{* 2}\left(v_{* 2}-1\right)\right], \quad \psi_{* 2}=\left[v_{* 2} \Lambda_{* 2} / k_{* 2}\right]
\end{gathered}
$$

\section{Construction of Design Matrix: Method I}

Consider a SBIB design $D$ with the parameters $(v, k, \lambda)$; each pair of treatments occurs together in $\lambda$ blocks. Take any pair of treatments, say, $(\theta, \phi)$ from this design. Then make two blocks from the design which contains the pair $(\theta, \phi)$.

1. Give the negative sign to the treatment $\theta$ and eliminate the treatment $\phi$ while the other $(k-2)$ remaining treatments of the same block remain as it is.

2. Give the negative sign to the treatment $\phi$ and eliminate the treatment $\theta$ while the other $(k-2)$ remaining treatments of the same block remain as it is.

The matrix $N_{1}$ of design $D_{1}$ is obtained. Now doing the same procedure for all possible $\left(\begin{array}{l}v \\ 2\end{array}\right)$ pairs of treatments, we obtain matrix $N_{i} ; i=1,2, \cdots,\left(\begin{array}{l}v \\ 2\end{array}\right)$. Then the incidence matrix $N_{* * 11}$ of the new design $D_{* * 1}$ so formed is the matrix having the elements $1,-1$ and 0 ; given as follows by juxtaposition 


$$
\left.N_{* * 1}=\left[\begin{array}{ccccccc}
N_{1} & \vdots & N_{2} & \vdots & \cdots & \vdots & N_{(} \\
2
\end{array}\right)\right]
$$

Then combining the incidence matrix $N$ of SBIB design repeated s-times with $N_{* * 1}$ we get the matrix $X$ of a chemical balance weighing design as

$$
X=\left[\begin{array}{ccc}
N_{* * 1} & \vdots & \overbrace{N \cdots N}^{s-t i m e s}
\end{array}\right]^{\prime}
$$

Under the present construction scheme, we have $n=2 \lambda\left(\begin{array}{l}v \\ 2\end{array}\right)+s b=\lambda v(v-1)+s b$ and $p=v$. Thus the each column of $X$ will contain $\delta_{1}=r(k-1)(k-2)+s r$ elements equal to $1, \delta_{2}=r(k-1)$ elements equal to -1 and $r(k-1)(v-k+1)$ elements equal to zero. Clearly such a design implies that each object is weighted $m=\delta_{1}+\delta_{2}=r(k-1)^{2}+s r$ times in $n=2 \lambda\left(\begin{array}{l}v \\ 2\end{array}\right)+s b$ weighing operations.

Lemma 3.4. A design given by $X$ of the form (8) is non singular if and only if $\lambda\left[v(k-3)-\left(k^{2}-6 \lambda+7\right)\right]+s(r-\lambda) \neq 0$.

Proof. For the design matrix $X$ given by (8), we have

$$
\begin{aligned}
X^{\prime} X & =[\{r(k-1)(k-3)+s r\}-\{\lambda(k-2)(k-5)+s \lambda\}] I_{v}+[\lambda(k-2)(k-5)+s \lambda] J_{v v} \\
& =\left[\lambda\left\{v(k-3)-\left(k^{2}-6 \lambda+7\right)\right\}+s(r-\lambda)\right] I_{v}+[\lambda\{(k-2)(k-5)+s\}] J_{v v}
\end{aligned}
$$

and

$$
\left|X^{\prime} X\right|=\left[r\left\{\left(k^{3}-7 k^{2}+13 k-7\right)+s k\right\}\right]\left[\lambda\left\{v(k-3)-\left(k^{2}-6 \lambda+7\right)\right\}+s(r-\lambda)\right]^{v-1}
$$

the determinant (10) is equal to zero if and only if

$$
\begin{aligned}
& r(k-1)(k-3)+s r=\lambda(k-2)(k-5)+s \lambda \\
\Rightarrow & \lambda\left[v(k-3)-\left(k^{2}-6 \lambda+7\right)\right]+s(r-\lambda)=0 \\
\text { or } \quad & r\left[\left(k^{3}-7 k^{2}+13 k-7\right)+s k\right]=0
\end{aligned}
$$

but $\left[r\left\{\left(k^{3}-7 k^{2}+13 k-7\right)+s k\right\}\right]$ is positive and then $\operatorname{det}\left(X^{\prime} X\right)=0$ if and only if $\lambda\left[v(k-3)-\left(k^{2}-6 \lambda+7\right)\right]+s(r-\lambda)=0$. So the lemma is proved.

Theorem 3.5. The non-singular chemical balance weighing design with matrix $X$ given by (8) is optimal if and only if

$$
\lambda[(k-2)(k-5)+s]=0 .
$$

Proof. From the conditions (5) and (9) it follows that a chemical balance weighing design is optimal if and only if the condition (11) holds. Hence the theorem.

If the chemical balance weighing design given by matrix $X$ of the form (8) is optimal then

$$
\operatorname{Var}\left(\hat{w}_{j}\right)=\frac{\sigma^{2}}{r\left[(k-1)^{2}+s\right]} ; j=1,2, \cdots, p
$$

Example 3.6. Consider a SBIB design with parameters $v=b=4, r=k=3, \lambda=2$; whose blocks are given by $(1,2,3),(1,2,4),(1,3,4),(2,3,4)$. 
Theorem 3.5 yields a design matrix $X$ of optimum chemical balance weighing design as

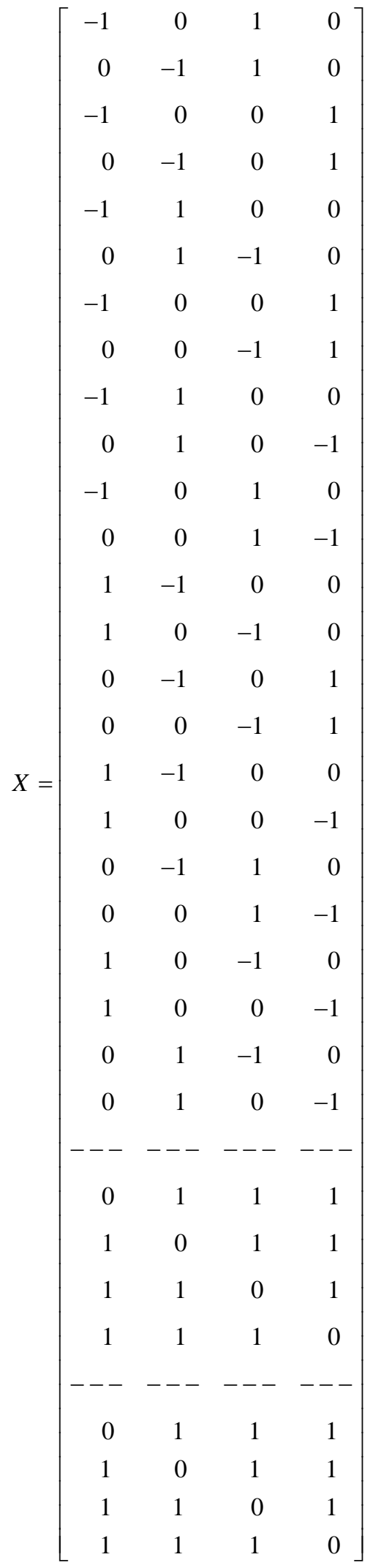


Clearly such a design implies that each object is weighted $m=18$ times in $n=32$ weighing operations and $\operatorname{Var}(\hat{w})=\sigma^{2} / 18$ for each $j=1,2,3,4$.

Corollary 3.7. If the SBIB design exists with parameters $(v, k, \lambda)$; then the design matrix $X$ so formed in (8) is optimum chemical balance weighing design iff $2<k<5$ and $s \leq 2$.

Remark: In SBIB design with parameters $(v, k, \lambda)$; if $k=0$ then the design matrix given in (8) is perfectly optimum and $s=0$ in this case.

Corollary 3.8. If in the design $D_{* * 1} ;-1$ is replaced by zero then the new design $D_{* * * 1}$ so formed is a BIB design with parameters $V=v, B=2\left(\begin{array}{l}v \\ 2\end{array}\right), R=r(k-1)(k-2), K=k-2, \Lambda=2 \lambda\left(\begin{array}{c}k-2 \\ 2\end{array}\right)$. Then the structure

$$
N^{* * 1}=\left[\begin{array}{lll}
N_{* * * 1} & \vdots & \overbrace{N \cdots N}^{s \text {-times }}
\end{array}\right]
$$

form a pairwise VB and EB design $D^{* * 1}$ with parameters

$v^{* * 1}=V, \quad b^{* * 1}=B+s b, \quad r^{* * 1}=R+s r, \quad k_{1}^{* * 1}=k-\lambda, \quad k_{2}^{* * 1}=k, \quad \lambda^{* * 1}=\Lambda+s \lambda$,

$\psi^{* * 1}=v\left[(k-\lambda-1)+\frac{s \lambda}{k}\right]$ and

$\mu^{* * 1}=1-\frac{v}{r^{* * 1}}\left[(k-\lambda-1)+\frac{s \lambda}{k}\right]$.

\section{Construction of Design Matrix: Method II}

Consider a SBIB design D with the parameters $(v, k, \lambda)$; each pair of treatments occurs together in $\lambda$ blocks. Take any pair of treatments, say, $(\theta, \phi)$ from this design. Then make three blocks from the design which contains the pair $(\theta, \phi)$.

1. Give the negative sign to the treatment $\theta$ and eliminate the treatment $\phi$ while the other $(k-2)$ remaining treatments of the same block remain as it is.

2. Give the negative sign to the treatment $\phi$ and eliminate the treatment $\theta$ while the other $(k-2)$ remaining treatments of the same block remain as it is.

3. Give the negative sign to both the treatments $\theta$ and $\phi$ while the other remaining treatments of the same block remain as it is.

The matrix $N_{1}$ of design $D_{1}$ is obtained. Now doing the same procedure for all possible $\left(\begin{array}{l}v \\ 2\end{array}\right)$ pairs of treatments, we obtain matrix $N_{i} ; i=1,2, \cdots,\left(\begin{array}{l}v \\ 2\end{array}\right)$. Then the incidence matrix $N_{* * 2}$ of the new design $D_{* * 2}$ so formed is the matrix having the elements $1,-1$ and 0 ; given as follows juxtaposition:

$$
N_{* * 2}=\left[\begin{array}{lllllll}
N_{1} & \vdots & N_{2} & \vdots & \cdots & \vdots & N_{(} \\
& & & & & &
\end{array}\right]
$$

Then combining the incidence matrix $N$ of SBIB design repeated s-times with $N_{* * 2}$ we get the matrix $X$ of a chemical balance weighing design as:

$$
X=\left[\begin{array}{ccc}
N_{* * 2} & \vdots & \overbrace{N \cdots N}^{s \text {-times }}
\end{array}\right]^{\prime}
$$

Under the present construction scheme, we have $n=3 \lambda\left(\begin{array}{l}v \\ 2\end{array}\right)+s b$ and $p=v$. Thus the each column of $X$ 
will contain $\delta_{1}=[\{3 r(k-1)(k-2) / 2\}+s r]$ elements equal to $1, \delta_{2}=2 r(k-1)$ elements equal to -1 and $n-\delta_{1}-\delta_{2}$ elements equal to zero. Clearly such a design implies that each object is weighted $m=\delta_{1}+\delta_{2}=\left[(3 k-2)\left(\begin{array}{l}r \\ 2\end{array}\right)\right]+s r$ times in $n=3 \lambda\left(\begin{array}{l}v \\ 2\end{array}\right)+s b$ weighing operations.

Lemma 4.9. A design given by $X$ of the form (14) is non singular if and only if $\left[(3 k-10)\left(\begin{array}{l}r \\ 2\end{array}\right)+s r\right] \neq\left[\frac{\lambda}{2}\left(3 k^{2}-23 k+36\right)+s \lambda\right]$.

Proof. For the design matrix $X$ given by (14), we have

$$
\begin{aligned}
X^{\prime} X & =\left[\left(\frac{r(k-1)(3 k-10)}{2}+s r\right)-\left(\frac{\lambda}{2}\left(3 k^{2}-23 k+36\right)+s \lambda\right)\right] I_{v}+\left[\frac{\lambda}{2}\left(3 k^{2}-23 k+36\right)+s \lambda\right] J_{v v} \\
& =\left[\left\{(3 k-10)\left(\begin{array}{l}
r \\
2
\end{array}\right)+s r\right\}-\left\{\frac{\lambda}{2}\left(3 k^{2}-23 k+36\right)+s \lambda\right\}\right] I_{v}+\left[\frac{\lambda}{2}\left(3 k^{2}-23 k+36\right)+s \lambda\right] J_{v v}
\end{aligned}
$$

and

$$
\begin{aligned}
\left|X^{\prime} X\right|= & {\left[\left\{(3 k-10)\left(\begin{array}{l}
r \\
2
\end{array}\right)+s r\right\}+(v-1)\left\{\frac{\lambda}{2}\left(3 k^{2}-23 k+36\right)+s \lambda\right\}\right] } \\
& \times\left[\left\{(3 k-10)\left(\begin{array}{l}
r \\
2
\end{array}\right)+s r\right\}-\left\{\frac{\lambda}{2}\left(3 k^{2}-23 k+36\right)+s \lambda\right\}\right]^{\nu-1}
\end{aligned}
$$

the determinant (16) is equal to zero if and only if

$$
(3 k-10)\left(\begin{array}{l}
r \\
2
\end{array}\right)+s r=\frac{\lambda}{2}\left(3 k^{2}-23 k+36\right)+s \lambda
$$

or $(3 k-10)\left(\begin{array}{l}r \\ 2\end{array}\right)+s r=(1-v) \frac{\lambda}{2}\left(3 k^{2}-23 k+36\right)+s \lambda$

but $\left[(3 k-10)\left(\begin{array}{l}r \\ 2\end{array}\right)+s r\right]+(v-1)\left\{\frac{\lambda}{2}\left(3 k^{2}-23 k+36\right)+s \lambda\right\}$ is positive and then $\operatorname{det}\left(X^{\prime} X\right)=0$ if and only if $(3 k-10)\left(\begin{array}{l}r \\ 2\end{array}\right)+s r=\frac{\lambda}{2}\left(3 k^{2}-23 k+36\right)+s \lambda$. So the lemma is proved.

Theorem 4.10. The non-singular chemical balance weighing design with matrix $X$ given by (14) is optimal if and only if

$$
\frac{\lambda}{2}\left(3 k^{2}-23 k+36\right)+s \lambda=0 .
$$

Proof. From the conditions (5) and (15) it follows that a chemical balance weighing design is optimal if and only if the condition (17) holds. Hence the theorem.

If the chemical balance weighing design given by matrix $X$ of the form (14) is optimal then

$$
\operatorname{Var}\left(\hat{w}_{j}\right)=\frac{\sigma^{2}}{\left\{(3 k-2)\left(\begin{array}{l}
r \\
2
\end{array}\right)+s r\right\}} ; j=1,2, \cdots, p
$$

Example 4.11. Consider a SBIB design with parameters $v=b=4, r=k=3, \lambda=2$; whose blocks are given by $(1,2,3),(1,2,4),(1,3,4),(2,3,4)$. 
Theorem 4.10 yields a design matrix $X$ of optimum chemical balance weighing design as

$$
X=\left[\begin{array}{cccc}
-1 & 0 & 1 & 0 \\
0 & -1 & 1 & 0 \\
-1 & -1 & 1 & 0 \\
-1 & 0 & 0 & 1 \\
0 & -1 & 0 & 1 \\
-1 & -1 & 0 & 1 \\
-1 & 1 & 0 & 0 \\
0 & 1 & -1 & 0 \\
-1 & 1 & -1 & 0 \\
-1 & 0 & 0 & 1 \\
0 & 0 & -1 & 1 \\
-1 & 0 & -1 & 1 \\
-1 & 1 & 0 & 0 \\
0 & 1 & 0 & -1 \\
-1 & 1 & 0 & -1 \\
-1 & 0 & 1 & 0 \\
0 & 0 & 1 & -1 \\
-1 & 0 & 1 & -1 \\
1 & -1 & 0 & 0 \\
1 & 0 & -1 & 0 \\
1 & -1 & -1 & 0 \\
0 & -1 & 0 & 1 \\
0 & 0 & -1 & 1 \\
0 & -1 & -1 & 1 \\
1 & -1 & 0 & 0 \\
1 & 0 & 0 & -1 \\
1 & -1 & 0 & -1 \\
0 & -1 & 1 & 0 \\
0 & 0 & 1 & -1 \\
0 & -1 & 1 & -1 \\
1 & 0 & -1 & 0 \\
1 & 0 & 0 & -1 \\
1 & 0 & -1 & -1 \\
0 & 1 & -1 & 0 \\
0 & 1 & 0 & -1 \\
0 & 1 & -1 & -1 \\
-- & -- & -- & -- \\
0 & 1 & 1 & 1 \\
1 & 0 & 1 & 1 \\
1 & 1 & 0 & 1 \\
1 & 1 & 1 & 0 \\
-- & -- & -- & -- \\
0 & 1 & 1 & 1 \\
1 & 0 & 1 & 1 \\
1 & 1 & 0 & 1 \\
1 & 1 & 1 & 0 \\
-- & -- & -- & -- \\
0 & 1 & 1 & 1 \\
1 & 0 & 1 & 1 \\
1 & 1 & 0 & 1 \\
1 & 1 & 1 & 0
\end{array}\right]
$$


Clearly such a design implies that each object is weighted $m=30$ times in $n=48$ weighing operations and $\operatorname{Var}(\hat{w})=\sigma^{2} / 30$ for each $j=1,2,3,4$.

Corollary 4.12. If the SBIB design exists with parameters $(v, k, \lambda)$; then the design matrix $X$ of the form (14) is optimum chemical balance weighing design iff $k \leq 5$ and $s \leq 4$.

Corollary 4.13. If in the design $D_{* * 2} ;-1$ is replaced by zero then the new design $D_{* * * 2}$ so formed is a BIB design with parameters $V=v, B=3\left(\begin{array}{l}v \\ 2\end{array}\right), R=[3 r(k-1)(k-2)] / 2, K=k-2, \quad \Lambda=3 \lambda\left(\begin{array}{c}k-2 \\ 2\end{array}\right)$. Then the structure

$$
N^{* * 2}=\left[\begin{array}{lll}
N_{* * * 2} & \vdots & \overbrace{N \cdots N}^{s-\text { times }}
\end{array}\right]
$$

form a pairwise VB and EB design $D^{* * 2}$ with parameters

$v^{* * 2}=v, \quad b^{* * 2}=B+s b, r^{* * 2}=R+s r, \quad k_{1}^{* * 2}=k-2, \quad k_{2}^{* * 2}=k, \lambda^{* * 2}=\Lambda+s \lambda$,

$\psi^{* * 2}=v \lambda\left[\frac{3(k-3)}{2}+\frac{s}{k}\right]$ and

$\mu^{* * 2}=1-\frac{v \lambda}{r^{* * 2}}\left[\frac{3(k-3)}{2}+\frac{s}{k}\right]$.

\section{Result and Discussion}

The following Table 1 and Table 2 provide the list of pairwise variance and efficiency balanced block designs for Methods I and II respectively, which can be obtained by using certain known SBIB designs.

Table 1. For Method I.

\begin{tabular}{ccccccccccc}
\hline S. No. & $v^{*+1}$ & $b^{*+1}$ & $r^{*+1}$ & $k_{1}^{*+1}$ & $k_{2}^{* * 1}$ & $\lambda^{*+1}$ & $\psi^{* * 1}$ & $\mu^{*+1}$ & $\begin{array}{c}\text { Reference } \\
\text { No. }\end{array}$ \\
\hline 1 & 5 & 70 & 32 & 2 & 4 & 12 & 22.5 & 0.2968 & $\mathrm{R}(4)$ \\
2 & 7 & 98 & 32 & 2 & 4 & 8 & 21 & 0.3437 & $\mathrm{R}(11)$ \\
3 & 13 & 182 & 32 & 2 & 4 & 4 & 19.5 & 0.3906 & $\mathrm{R}(37), \mathrm{MH}(3)$ \\
\hline
\end{tabular}

Table 2. For Method II.

\begin{tabular}{ccccccccccc}
\hline S. No. & $v^{* * 2}$ & $b^{* 2}$ & $r^{*+2}$ & $k_{1}^{*+2}$ & $k_{2}^{*+2}$ & $\lambda^{*+2}$ & $\psi^{* * 2}$ & $\mu^{* * 2}$ & $\begin{array}{c}\text { Reference } \\
\text { No. }\end{array}$ \\
\hline 1 & 5 & 110 & 32 & 2 & 4 & 12 & 22.5 & 0.2968 & $\mathrm{R}(4)$ \\
2 & 7 & 84 & 12 & 1 & 3 & 2 & 4.667 & 0.6111 & $\mathrm{R}(10), \mathrm{MH}(1)$ \\
3 & 7 & 154 & 32 & 2 & 4 & 8 & 21 & 0.3437 & $\mathrm{R}(11)$ \\
4 & 13 & 286 & 32 & 2 & 4 & 4 & 19.5 & 0.3906 & $\mathrm{R}(37), \mathrm{MH}(3)$ \\
\hline
\end{tabular}

${ }^{* * *}$ The symbols $\mathrm{R}(\alpha)$ and $\mathrm{MH}(\alpha)$ denote the reference number $\alpha$ in Raghavrao [30] and Marshal Halls [31] list.

\section{Conclusion}

In this research, we have significantly shown that the obtained designs are pairwise balanced as well as efficiency balanced. The only limitation of this research is that the obtained pairwise balanced designs are all have large number of replications.

\section{Acknowledgements}

We are grateful to the anonymous referees for their constructive comments and valuable suggestions. 


\section{References}

[1] Yates, F. (1935) Complex Experiments. Journal of the Royal Statistical Society, 2, 181-247. http://dx.doi.org/10.2307/2983638

[2] Hotelling, H. (1944) Some Improvements in Weighing and Other Experimental Techniques. Annals of Mathematical Statistics, 15, 297-306. http://dx.doi.org/10.1214/aoms/1177731236

[3] Banerjee, K.S. (1948) Weighing Designs and Balanced Incomplete Blocks. Annals of Mathematical Statistics, 19, 394399. http://dx.doi.org/10.1214/aoms/1177730204

[4] Banerjee, K.S. (1975) Weighing Designs for Chemistry, Medicine, Economics, Operations Research, Statistics. Marcel Dekker Inc., New York.

[5] Dey, A. (1969) A Note on Weighing Designs. Annals of the Institute of Statistical Mathematics, 21, 343-346. http://dx.doi.org/10.1007/BF02532262

[6] Dey, A. (1971) On Some Chemical Balance Weighing Designs. Aust. J. Stat., 13, 137-141. http://dx.doi.org/10.1111/j.1467-842X.1971.tb01252.x

[7] Raghavarao, D. (1959) Some Optimum Weighing Designs. Annals of Mathematical Statistics, 30, 295-303. http://dx.doi.org/10.1214/aoms/1177706253

[8] Ceranka, B. and Graczyk, M. (2001) Optimum Chemical Balance Weighing Designs under the Restriction on the Number in Which Each Object Is Weighed. Discussiones Mathematica Probability and Statistics, 21, 113-120.

[9] Ceranka, B. and Graczyk, M. (2002) Optimum Chemical Balance Weighing Designs Based on Balanced Incomplete Block Designs and Balanced Bipartite Block Designs. Mathematica, 11, 19-27.

[10] Ceranka, B. and Graczyk, M. (2004) Ternary Balanced Block Designs Leading to Chemical Balance Weighing Designs For v + 1 Objects. Biometrica, 34, 49-62.

[11] Ceranka, B. and Graczyk, M. (2010) Some Construction of Optimum Chemical Balance Weighing Designs. Acta Universitatis Lodziensis, Folia Economic, 235, 235-239.

[12] Awad, R. and Banerjee, S. (2013) Some Construction Methods of Optimum Chemical Balance Weighing Designs I. Journal of Emerging Trends in Engineering and Applied Sciences (JETEAS), 4, 778-783.

[13] Awad, R. and Banerjee, S. (2014) Some Construction Methods of Optimum Chemical Balance Weighing Designs II. Journal of Emerging Trends in Engineering and Applied Sciences (JETEAS), 5, 39-44.

[14] Awad, R. and Banerjee, S. (2014) Some Construction Methods of A-Optimum Chemical Balance Weighing Designs. Journal of Applied Mathematics and Physics (JAMP), 2, 1159-1170. http://dx.doi.org/10.4236/jamp.2014.213136

[15] Billington, E.J.B. and Robinson, P.T. (1983) A List of Balanced Ternary Designs with $\mathrm{R} \leq 15$ and Some Necessary Existence Conditions. Ars Combinatoria, 10, 235-258.

[16] Angelis, L., Moyssiadis, C. and Kageyama, S. (1994) Constructions of Generalized Binary Proper Efficiency Balanced Block Designs with Two Different Replication Numbers. Sankhya, 56, 259-266.

[17] Caliski, T. (1977) On the Notation of Balance Block Designs. In: Barra, J.R., Brodeau, F., Romier, G. and Van Cutsem, B., Eds., Recent Developments in Statistics, North-Holland Publishing Company, Amsterdam, 365-374.

[18] Puri, P.D. and Nigam, A.K. (1975) On Patterns of Efficiency Balanced Designs. Journal of the Royal Statistical Society, B37, 457-458.

[19] Rao, V.R. (1958) A Note on Balanced Designs. The Annals of Mathematical Statistics, 29, 290-294. http://dx.doi.org/10.1214/aoms/1177706729

[20] Caliski, T. (1971) On Some Desirable Patterns in Block Designs. Biometrika, 27, 275-292. http://dx.doi.org/10.2307/2528995

[21] Caliski, T. and Kageyama, S. (2003) Block Designs: A Randomized Approach, Volume I: Analysis. Springer, New York.

[22] Caliski, T. and Kageyama, S. (2003) Block Designs: A Randomized Approach, Volume II: Design. Springer, New York.

[23] Kageyama, S. (1980) On Properties of Efficiency-Balanced Designs. Communications in Statistics-Theory and Methods, 9, 597-616.

[24] Preece, D.A. (1967) Nested Balanced Incomplete Block Designs. Biometrika, 54, 479-486. http://dx.doi.org/10.1093/biomet/54.3-4.479

[25] Ghosh, D.K. and Karmakar, P.K. (1988) Some Series of Efficiency Balanced Designs. Australian Journal of Statistics, 30, 47-51. http://dx.doi.org/10.1111/j.1467-842X.1988.tb00610.x

[26] Ghosh, D.K., Shah, A. and Kageyama, S. (1994) Construction of Variance Balanced and Efficiency Balanced Block 
Designs. Journal of the Japan Statistical Society, 24, 201-208.

[27] Banerjee, S. (1985) Some Combinatorial Problems in Incomplete Block Designs. Unpublished PhD Thesis, Devi Ahilya University, Indore.

[28] Banerjee, S., Kageyama, S. and Rai, S. (2003) Nested Balanced Equireplicate Block Designs. Discussion Mathematicae: Probability and Statistics, 23, 45-68.

[29] Banerjee, S., Kageyama, S. and Rudra, S. (2005) Constructions of Nested Pairwise Efficiency and Variance Balanced Designs. Communications in Statistics-Theory and Methods, 37, 1571-1578.

[30] Raghavarao, D. (1971) Constructions and Combinatorial Problems in Designs of Experiments. John Wiley, New York.

[31] Hall Jr., M. (1986) Combinatorial Theory. John Wiley, New York. 